Experi ment al evi dence of the one-thi $r d$ magnet i zat $i$ on $p l$ at eau i $n$ the di anond chai $n$ compound $\mathrm{Cu} 3(\mathrm{CO} 3) 2(\mathrm{OH}) 2$

\begin{tabular}{|l|l|}
\hline 著者 & $\begin{array}{l}\text { K KUCH H, FUJI I Y, CH BA M M TSUDO S, } \\
\text { I DEHARA T, KUMAl T }\end{array}$ \\
\hline $\begin{array}{l}\text { j our nal or } \\
\text { publ i cat i on ti t l e }\end{array}$ & Jour nal of Nagnet i sm and Nagnet i c Nat er i al s \\
\hline vol une & $272-276$ \\
\hline number & 2 \\
\hline page r ange & $900-901$ \\
\hline year & 2004 05 \\
\hline URL & ht t p: //hdl . handl e. net /10098/1612 \\
\hline
\end{tabular}




\title{
Experimental evidence of the one-third magnetization plateau in the diamond chain compound $\mathrm{Cu}_{3}\left(\mathrm{CO}_{3}\right)_{2}(\mathrm{OH})_{2}$
}

\author{
H. Kikuchi ${ }^{a}$, Y. Fujii ${ }^{a}$, M. Chiba ${ }^{a}$, S. Mitsudo ${ }^{b}$, T. Idehara ${ }^{b}$, T. Kuwai ${ }^{c}$ \\ ${ }^{a}$ Department of Applied Physics, Fukui University, Fukui 910-8507, Japan \\ ${ }^{b}$ Research Center for Development of Far-Infrared Region, Fukui University, Fukui 910-8507, Japan \\ 'Department of Physics, Toyama University, Toyama 930-8555, Japan
}

\begin{abstract}
The magnetic susceptibility, high-field magnetization and specific heat of $\mathrm{Cu}_{3}\left(\mathrm{CO}_{3}\right)_{2}(\mathrm{OH})_{2}$, an actual material for the frustrating diamond chain spin model, have been measured using single crystals. Two broad peaks were observed at around 22 and $5 \mathrm{~K}$ both in the magnetic susceptibility and the specific heat. The magnetization curve had a definite plateau at one-third of the saturation magnetization.
\end{abstract}

Keywords: Frustration; One-dimensional antiferromagnet; High-field magnetization; $\mathrm{Cu}_{3}\left(\mathrm{CO}_{3}\right)_{2}(\mathrm{OH})_{2}$

\section{Introduction}

The influence of the spin frustration on a ground state of a quantum spin system is of great interest and has been studied actively. One of the most simple frustrated quantum system is the so-called diamond chain [1] in which diamond-shaped units compose one-dimensional lattice. Recent theoretical investigation [2] on the distorted diamond chain model determined the ground state phase diagram which is composed of the dimerized. spin fluid and ferrimagnetic phases. The calculated magnetization curve at $T=0$ was found to have $\frac{1}{3}$ plateau where the magnetizaiton is quantized. There has been, however, no experimental report on an experimental observation of this $\frac{1}{3}$ plateau.

We have already reported [3] that a compound $\mathrm{Cu}_{3}\left(\mathrm{CO}_{3}\right)_{2}(\mathrm{OH})_{2}$ (azurite), in which $\mathrm{Cu}^{2+}\left(\mathrm{S}=\frac{1}{2}\right.$ ) monomers and dimers are arranged along the crystallographic $b$-axis to make an infinite chain, is regarded as an actual model material for the distorted diamond chain. In order to verify the theoretical expectation, we have measured magnetic susceptibility, high-field magnetization and specific heat on a natural mineral single crystal of $\mathrm{Cu}_{3}\left(\mathrm{CO}_{3}\right)_{2}(\mathrm{OH})_{2}$.

\section{Experimental details}

The single crystal samples of azurite were purchased at a stone shop. Quality of the sample was checked by a powder $X$-ray diffraction. The magnetic susceptibility was measured using Quantum Design Physical Property Measurement System (PPMS) in the temperature range $2-300 \mathrm{~K}$. The magnetization curve up to $38 \mathrm{~T}$ was measured using pulsed magnet. The specific heat was measured above $0.33 \mathrm{~K}$ by quasi-adiabatic method.

\section{Results and discussion}

Fig. 1 shows the temperature dependence of the magnetic susceptibility. The magnetic field of 1000 Oe was applied parallel or perpendicular to the $b$-axis of the 


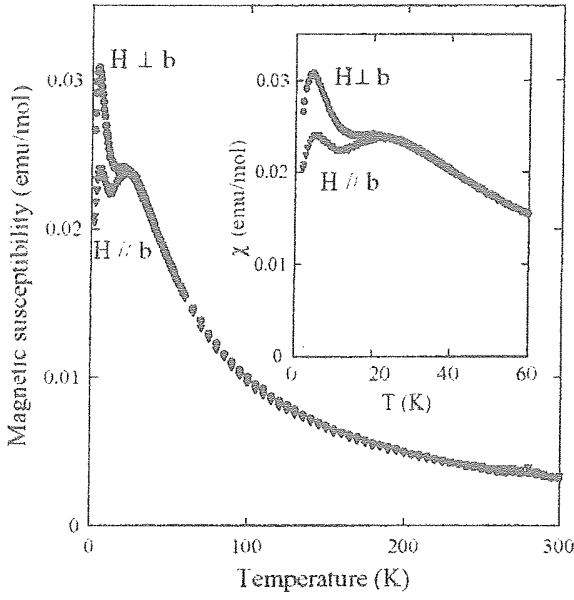

Fig. 1. The temperature dependence of the magnetic susceptibility of a single crystal of $\mathrm{Cu}_{3}\left(\mathrm{CO}_{3}\right)_{2}(\mathrm{OH})_{2}$ measured in field of 1000 oe applied parallel or perpendicular to the $b$-axis. Inset shows the low-temperature part of the magnetic susceptibility.

single crystal. Two broad peaks were observed at around 22 and $5 \mathrm{~K}$. The specific heat also had two maxima at these temperatures. These broad peaks suggest the two stage development of short-range magnetic ordering.

Fig. 2 shows the magnetization curve of $\mathrm{Cu}_{3}\left(\mathrm{CO}_{3}\right)_{2}(\mathrm{OH})_{2}$ for $H|| b$. No hysteresis was observed on increasing and decreasing the applied magnetic field. Successive transitions were observed at critical helds of $H_{c 1}^{\|}=16 \mathrm{~T}, H_{c 2}^{\|\|}=26 \mathrm{~T}$ and $H_{c 3}^{\|}=32.5 \mathrm{~T}$. $H_{c 3}^{\| l}$ is a saturation field. The magetization kept almost constant value of $\frac{1}{3}$ of the saturation moment in the field region between $H_{\mathrm{c} 1}^{\|}$and $H_{\mathrm{c} 2}^{\|}$. This is the first experimental observation of the $\frac{1}{3}$ plateau in the diamond chain magnet, which was expected theoretically. The $\frac{1}{3}$ platea was also observed when the magnetic field was applied

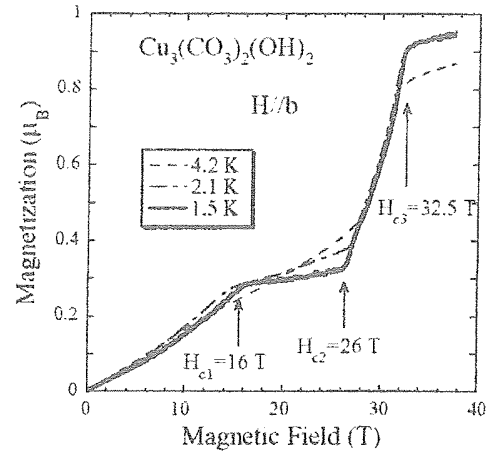

Fig. 2. Magnetization curve of $\mathrm{Cu}_{3}\left(\mathrm{CO}_{3}\right)_{2}(\mathrm{OH})_{2}$ for $\mathrm{H} \| b$ up to $38 T$ at various temperatures.

perpendicular to the $b$-axis with critical fields of $H H_{\mathrm{cl}}=$ $11 \mathrm{~T}, H_{c 2}^{\frac{1}{2}}=30 \mathrm{~T}$ and $H_{\mathrm{c} 3} \frac{1}{3}=32.5 \mathrm{~T}$. The plateau region was found to be broader for $H \perp b$ than $H \| b$. An origin of this anisotropic behavior is not clear at this time.

\section{Aclnowledigements}

This work is supported by Grant-in-Aid for Scientific Research on Priority Areas (B) (No. 13130204) from the Ministry of Educations. Culture, Sports, Science and Technology, Japan.

\section{Teferences}

[1] K. Takano, K. Kubo, H. Sakamoto, I. Phys.: Condens. Matter 8 (1996) 6405

[2] T. Tonegawa, K. Okamoto, T. Hikihara, Y. Takahashi, M. Kaburagi, J. Phys. Soc. Jpn. 69 (Suppl: A) (2000) 332.

[3] H. Kikuchi, et al., Physica B 329-333 (2003) 967. 